\title{
Dosimetric evaluation of four whole brain radiation therapy approaches with hippocampus and inner ear avoidance and simultaneous integrated boost for limited brain metastases
}

\author{
Aijun Jiang ${ }^{1,2+}$, Weipeng Sun ${ }^{2 \dagger}$, Fen Zhao ${ }^{2}$, Zhenxuan $\mathrm{Wu}^{2}$, Dongping Shang ${ }^{2}$, Qingxi $\mathrm{Yu}^{2}$, Suzhen Wang ${ }^{2}$, \\ Jian Zhu' ${ }^{2}$ Fengchang Yang ${ }^{2}$ and Shuanghu Yuan ${ }^{2 *}$
}

\begin{abstract}
Aims: To perform a dosimetric evaluation of four different simultaneous integrated boost whole brain radiotherapy modalities with hippocampus and inner ear avoidance in the treatment of limited brain metastases.

Methods: Computed tomography/magnetic resonance imaging data of 10 patients with limited (1-5) brain metastases were used to replan step-and-shoot intensity-modulated radiotherapy (sIMRT), dynamic intensitymodulated radiation therapy (dIMRT), volumetric-modulated arc therapy (VMAT), and helical tomotherapy (Tomo). The prescribed doses of 40-50 Gy in 10 fractions and $30 \mathrm{~Gy}$ in 10 fractions were simultaneously delivered to the metastatic lesions and the whole-brain volume, respectively. The hippocampal dose met the RTOG 0933 criteria for hippocampal avoidance (Dmax $\leq 17 \mathrm{~Gy}, \mathrm{D} 100 \% \leq 10 \mathrm{~Gy}$ ). The inner ear dose was restrained to Dmean $\leq 15 \mathrm{~Gy}$. Target coverage (TC), homogeneity index $(\mathrm{HI})$, conformity index $(\mathrm{Cl})$, maximum dose (Dmax), minimum dose (Dmin) and dose to organs at risk (OARs) were compared.
\end{abstract}

Results: All plans met the indicated dose restrictions. The mean percentage of planning target volume of metastases (PTVmets) coverage ranged from 97.1 to $99.4 \%$. For planning target volume of brain (PTVbrain), Tomo provided the lowest average D2\% (37.5 $\pm 2.8 \mathrm{~Gy})$, the highest average D98\% (25.2 $\pm 2.0 \mathrm{~Gy})$, and the best TC (92.6\% $\pm 2.1 \%)$ and $\mathrm{Cl}(0.79 \pm 0.06)$. The two fixed gantry IMRT modalities (step and shot, dynamic) provided similar PTVbrain dose homogeneity (both 0.76 ). Significant differences across the four approaches were observed for the maximum and minimum doses to the hippocampus and the maximum doses to the eyes, lens and optic nerves.

Conclusion: All four radiotherapy modalities produced acceptable treatment plans with good avoidance of the hippocampus and inner ear. Tomo obtained satisfactory PTVbrain coverage and the best homogeneity index.

Trial registration: Clinicaltrials.gov, NCT03414944. Registered 29 January 2018

Keywords: Brain metastases, Dosimetry, Hippocampus, Inner ear, Intensity-modulated radiation therapy, Volumetricmodulated arc therapy, Tomotherapy

\footnotetext{
* Correspondence: yuanshuanghu@sina.com

${ }^{\dagger}$ Aijun Jiang and Weipeng Sun contributed equally to this work.

${ }^{2}$ Department of Radiation Oncology, Shandong Cancer Hospital affiliated to

Shandong University, 440 Jiyan Road, Jinan 250117, Shandong, China

Full list of author information is available at the end of the article
}

(c) The Author(s). 2019 Open Access This article is distributed under the terms of the Creative Commons Attribution 4.0 International License (http://creativecommons.org/licenses/by/4.0/), which permits unrestricted use, distribution, and reproduction in any medium, provided you give appropriate credit to the original author(s) and the source, provide a link to the Creative Commons license, and indicate if changes were made. The Creative Commons Public Domain Dedication waiver (http://creativecommons.org/publicdomain/zero/1.0/) applies to the data made available in this article, unless otherwise stated. 


\section{Introduction}

Brain metastases occur in $20-40 \%$ of cancer patients [1], and with improvements in local control and new effective systemic treatments, this incidence continues to increase [2]. The overall prognosis of cancer cases with brain metastases is poor. Whole brain radiotherapy (WBRT) is a fundamental radiation modality used specifically for patients with extensive brain metastases [3-7]. However, WBRT is associated with a short local control time and side effects including neurocognition dysfunction [8] and hearing deficits [9]. It is known that radiation-induced damage to the hippocampus plays an important role in the cognitive dysfunction $[4,8,10-$ 12], and hearing impairment can be linked to damage to the inner ear [13]. The threshold cochlear dose for hearing loss with cisplatin-based chemotherapy and radiotherapy was predicted to be $10 \mathrm{~Gy}$ [14]. Encouragingly, the results of the RTOG 0933 trial have shown that hippocampus avoidance can effectively reduce the cognitive impairment caused by WBRT [8].

WBRT with simultaneous integrated boost (SIB) for brain metastases has been proven to be advantageous in prolonging local control time and overall survival (OS) with a short treatment time $[15,16]$. With the development of advanced radiation technologies such as step-andshoot intensity-modulated radiotherapy (sIMRT), dynamic intensity-modulated radiation therapy (dIMRT), volumetric-modulated arc therapy (VMAT), and helical tomotherapy (Tomo), it is possible to perform WBRT with organs at risk (OARs) avoidance and SIB for brain metastases. Ferro $M$ et al. suggested that IMRT could deliver a SIB-WBRT treatment in patients with more than 3 brain metastases [17]. Sumit Sood et al. demonstrated the feasibility of WBRT using VMAT not only to spare hippocampus, but also significantly reduce dose to OARs, including the scalp, ear canal, cochleae, and parotid glands [18]. Tomo provides a homogeneous dose distribution to the whole brain and conformally avoids the hippocampus in a single treatment plan [19]. However, it is not clear which is the most appropriate WBRT approach with hippocampus and inner ear avoidance and simultaneous integrated boost for limited brain metastases. In the present study, a dosimetric comparison between sIMRT, dIMRT, VMAT and Tomo was performed to address the technical advantages of the four treatment modalities.

\section{Materials and methods}

\section{Simulation and contouring}

Ten consecutive patients with 1-5 brain metastases confirmed by magnetic resonance imaging (MRI), who were treated between September 2017 and October 2017, were included. All patients had histologic evidence of non-small cell lung cancer (NSCLC). All patients were in supine position wearing a thermoplastic mask (CIVCO, Orange City,
IA, USA) before computed tomography (CT) simulation. CT scans of the entire head region were acquired using a large Bore CT simulator (Philips, Cleveland, OH, USA) with a slice thickness of $1.5 \mathrm{~mm}$. The 3D e-THRIVE brain MRI axial T1-weighted sequences and double gadolinium contrast-enhanced sequences were obtained with a Philips Ingenia 3.0T MRI scanner (Philips, Cleveland, OH, USA) after CT simulation scanning. The slice thickness for MRI scans was $1.5 \mathrm{~mm}$. The CT and MRI datasets were registered in the Eclipse Version 11.0 planning system (Varian Medical Systems, Palo Alto, CA, USA) for target volume delineation.

Contouring was made using $2 \mathrm{D}$ brush on axial images. The gross tumor volume (GTV) was defined as the contrast-enhanced lesion. The planning tumor volume for metastases (PTVmets) was generated by adding to the GTV a $3 \mathrm{~mm}$ margin to correct for positional inaccuracies. The whole brain volume was contoured as the clinical target volume (CTV). The hippocampus was contoured on the MRI axial T1-weighted image sequence according to the RTOG 0933 protocol [8]. The hippocampus with a $5 \mathrm{~mm}$ expansion was defined as the area for hippocampal avoidance. The inner ear (cochlea, vestibule, semicircular canal) was delineated on CT bone window image datasets. To construct the planning target volume for brain (PTVbrain), a $3 \mathrm{~mm}$ margin was added to the CTV and adjusted to not overlap the inner ear volume. Then, the area for hippocampal avoidance and the GTV volume plus $5 \mathrm{~mm}$ (area for dose fall-off) were subtracted from the whole brain volume. Other OARs were outlined, including optic nerves, eyes, lenses, and brainstem. All contours were delineated by the same radiation oncologist and peer-reviewed.

\section{Prescription and planning}

The prescription dose was $45-50$ Gy in 10 fractions to the PTVmets and $30 \mathrm{~Gy}$ in 10 fractions to the PTVbrain simultaneously. The $30 \mathrm{~Gy}$ in 10 fractions scheme is a common scheme used in WBRT [1, 20]. The equivalent dose in 2 Gy/fraction (EQD2) of our fractionation scheme for PTVmets was calculated to be 54.4-62.5 $\mathrm{Gy}_{10}$ based on the LQ model [21]. All plans aimed to encompass at least $95 \%$ of PTVmets by $100 \%$ of the prescribed dose.

We constrained the unilateral or bilateral hippocampi (subgranular zone) dose according to findings of the RTOG 0933 trial [8]. The maximum dose (Dmax) and minimum dose (D100\%) limit for the hippocampal structures were $17 \mathrm{~Gy}$ and $10 \mathrm{~Gy}$ in 10 fractions, respectively. In two cases the metastasis was located close to the ipsilateral hippocampus $(<1.5 \mathrm{~cm})$, we met the dosimetric constraint criteria for the contralateral hippocampus. The mean dose (Dmean) constraints for inner ear structures were $15 \mathrm{~Gy}$ in 10 fractions. For other OARs, the 
maximal doses allowed were 9 Gy in 10 fractions for lens, $37.5 \mathrm{~Gy}$ in 10 fractions for brainstem and optic nerves, and $40 \mathrm{~Gy}$ in 10 fractions for normal brain. PTVmets coverage was the first aim in the planning process. Achieving homogenous and conformal PTVbrain dose distribution without exceeding the dose constraints of OARs was the second aim. The PTVbrain coverage could be reduced to $95 \%$ or even $<90 \%$ to meet the restrictions to the OARs.

For each case, three separate treatment plans (sIMRT, dIMRT, and VMAT) were optimized with $6 \mathrm{MV}$ photon beams for a linear accelerator (Trilogy, Varian Medical Systems) in the Eclipse Version 11.0 planning system. This linear accelerator is equipped with Millenium 120 multi-leaf collimator. The width of the central leaf pairs is $0.5 \mathrm{~cm}$. A Analytical Anisotropic Algorithm algorithm was used for dose calculation with a spatial resolution of $2.5 \mathrm{~mm}$. Beam arrangement for sIMRT and dIMRT was done using nine coplanar fields with a collimator angle of $0^{\circ}$. Two $360^{\circ}$ non-coplanar full arcs were used in the VMAT plans. Tomo planning was performed using the Tomotherapy treatment planning system (Accuray ${ }^{\circ}$ Planning Station, Hi Art $^{\circ}$ Version 5.1.3, Madison, WI, USA). Compared to $2.5 \mathrm{~cm}, 1.0 \mathrm{~cm}$ field width significantly improved the the whole brain homogeneity by $32 \%$ [18]. So, The field width and pitch were chosen as $1.05 \mathrm{~cm}$ and $0.5 \mathrm{~cm}$, respectively. Each plan was calculated to achieve the optimal deliverable plan with an acceptable target coverage while not exceeding the dose constraints to OARs. All plans were designed by the same medical physicist who had 5 years of planning experience and reviewed by other experts.

\section{Dosimetric evaluation}

Plans were finally reviewed and analyzed based on dosevolume histogram $(\mathrm{DVH})$. For each patient, the target coverage (TC), conformity index (CI), and homogeneity index (HI) for PTVmets and PTVbrain were evaluated. Subsequently, the following dose-volume parameters for OARs were calculated: 1) hippocampal maximum dose, 2) hippocampal mean dose, 3) hippocampal minimum dose, 4) inner ear mean dose, 5) eyes maximum dose, 6) lens maximum dose, 7) optic nerve maximum dose, and 8) brainstem maximum dose.

According to the conformity index reported by Paddick [22], the CI was defined as:

$$
C I=\frac{V^{2} \text { Tpres }}{T V \times V_{\text {pres }}}
$$

Where $V_{\text {Tpres }}$ represents the volume within the target receiving a dose greater than or equal to the prescription dose. $T V$ represents the target volume. $V_{\text {pres }}$ represents the volume receiving a dose greater than or equal to the prescription dose. The value of CI can range from 0 to 1 , and values closer to 1.0 are usually considered optimal.

The HI was defined as:

$$
H I=\frac{D_{2 \%}-D_{98 \%}}{D_{\text {median }}}
$$

This is the most commonly used formula in the literature [23], where $D_{2 \%}$ represents the maximum dose delivered to $2 \%$ of the target volume and $D_{98 \%}$ represents the minimum dose delivered to $98 \%$ of the target volume. $D_{\text {median }}$ represents the median dose to the target volume. $\mathrm{HI}$ is an objective tool to indicate the uniformity of dose distribution, and values close to 0 are considered optimal.

TC was defined as:

$$
T C=\frac{V_{T p r e s}}{T V} \times 100 \%
$$

$T C$ describes the fraction of the target volume receiving at least the prescription dose. For perfect TV coverage, TC equals $100 \%$.

\section{Statistical analysis}

Statistical comparisons among results obtained with the four treatment plan modalities were performed in SPSS, version 18.0 (SPSS Inc., Chicago, IL, USA) using oneway analysis of variance (ANOVA) with Tukey's multiple comparison post-hoc tests. All statistical tests were twosided, and differences were considered to be statistically significant if $p<0.05$.

\section{Results}

\section{Patient characteristics}

The characteristics of the patients are summarized in Table 1 . The 10 patients had a total of 26 metastases with a mean GTV of $12.8 \mathrm{cc}$ (range, 1.5-79.4 cc). The lesion diameters ranged from $1.2 \mathrm{~cm}$ to $6.4 \mathrm{~cm}$. The mean right and left hippocampus volumes were $2.97 \mathrm{cc}$ (range, $2.1-4.4 \mathrm{cc}$ ) and $2.65 \mathrm{cc}$ (range, 2.3-3.3 cc), respectively. In eight patients, lesions were located $1.5 \mathrm{~cm}$ away from the hippocampus. However, A metastatic lesion was in close proximity to or involved the hippocampus in two patients. The whole brain volume ranged from $1145.1 \mathrm{cc}$ to $1656.0 \mathrm{cc}$. The prescription dose was $45-50 \mathrm{~Gy}$ in 10 fractions for PTVmets. According to the biological equivalent dose (BED) formula, the EQD2 for PTVmets prescription dose ranged from 54.4 Gy to $62.5 \mathrm{~Gy}$.

\section{Dose distribution}

The dose parameters in the 10 patients are presented as mean values \pm standard deviation (SD) in Tables 2 and 3 . The prescribed dose was $45-50$ Gy in 10 fractions for the PTVmets and $30 \mathrm{~Gy}$ in 10 fractions for PTVbrain 
Table 1 Characteristics of 10 NSCLC patients with brain metastases

\begin{tabular}{llllll}
\hline Patient No. & Number of metastases & Total GTV volume $(\mathrm{cc})$ & Bilateral hippocampus volume $(\mathrm{cc})$ & Bilateral inner ear volume (cc) & Brain volume (cc) \\
\hline 1 & 2 & 3.7 & 6.3 & 2.9 & 1189.6 \\
2 & 1 & 12.9 & 5.7 & 2.0 & 1339.6 \\
3 & 1 & 6.4 & 4.4 & 2.6 & 1656.0 \\
4 & 4 & 9.0 & 5.8 & 2.0 & 1303.4 \\
5 & 3 & 3.2 & 4.7 & 2.6 & 1239.4 \\
6 & 1 & 7.3 & 5.0 & 2.3 & 1478.3 \\
7 & 2 & 79.4 & $2.2(\mathrm{R})$ & 1.8 & 1335.7 \\
8 & 3 & 1.5 & 6.3 & 2.0 & 1483.8 \\
9 & 4 & 1.6 & 7.7 & 2.3 & 1387.2 \\
10 & 5 & 3.3 & $2.7(\mathrm{~L})$ & 2.3 & 1145.1
\end{tabular}

GTV Gross tumor volume, $R$ right, $L$ left

simultaneously. Figure 1 shows a color wash representation of the dose distributions for the four treatment modalities from one representative case in our study. Figure 2 shows DVHs for the four treatment modalities from the same case.

\section{Dose for PTVmets}

PTVmets dose parameters (mean \pm SD) for the different modalities are presented along with the results of the statistical analysis in Table 2. The dose constraint was chosen as $100 \%$ of the prescription dose covering $95 \%$ of PTVmets. The D2\%, D98\%, Dmedian, TC, HI, and CI for PTVmets were compared. The mean percentage of PTVmets TC ranged from 97.1 to $99.4 \%$. The HI value for VMAT was highest among those of the tested modalities $(p<0.05)$. The four planning modalities provided similar mean CIs (range, 0.71-0.74), and no significant differences in the D2\%, D98\%, and Dmedian were found across the modalities.

\section{Dose for PTVbrain}

The results of the statistical dose evaluation for PTVbrain using sIMRT, dIMRT, VMAT and Tomo are shown in Table 3. Comparing PTVbrain dose distribution across modalities, Tomo provided the lowest average D2\%
(37.5 $\pm 2.8 \mathrm{~Gy})$ and the highest average D98\% (25.2 $\pm 2.0 \mathrm{~Gy})$. According to the HI formula, the best target dose homogeneity would be achieved by Tomo $(0.39, p<0.05)$, followed by VMAT $(0.57, \mathrm{p}<0.05)$. The two fixed gantry IMRT modalities (step and shot, dynamic) provided similar dose homogeneity (both 0.76). Although special care was taken to ensure that there was no dose larger than $120 \%$ of the prescription dose within the PTVbrain, some hotspots (Dmax > 36 Gy) were still found with the four planning modalities. These were considered acceptable though due to their small volume which limits their clinical significance (not D2\%). No statistically significant differences in $\mathrm{TC}$ and $\mathrm{CI}$ were found across modalities.

\section{Hippocampus and inner ear sparing}

The dose parameters of hippocampus and inner ear are compared in Table 4. All plans met the RTOG 0933 protocol dose compliance criteria for hippocampal sparing. Significant differences in Dmax and Dmin were found across the four planning modalities $(\mathrm{p}<0.05)$. There are no statistical significance for mean doses between sIMRT, dIMRT, VMAT and Tomo. Compared to VMAT and Tomo, sIMRT and dIMRT yeilded lower average maximum doses to the hippocampus. The

Table 2 Average dose parameters for PTVmets according to the four planning modalities

\begin{tabular}{llllll}
\hline & SIMRT & dIMRT & VMAT & Tomo & $p$ value \\
\hline D2\% (Gy) & $50.8 \pm 2.1$ & $50.9 \pm 2.4$ & $51.4 \pm 2.7$ & $50.2 \pm 2.7$ & $>0.05$ \\
D98\% (Gy) & $47.3 \pm 2.5$ & $47.6 \pm 2.4$ & $46.8 \pm 2.2$ & $46.9 \pm 2.3$ & $>0.05$ \\
Dmedian (Gy) & $49.7 \pm 2.5$ & $49.7 \pm 2.5$ & $49.7 \pm 2.5$ & $9.3 \pm 2.6$ & $>0.05$ \\
TC (\%) & $99.1 \pm 1.0$ & $99.4 \pm 0.7$ & $98.9 \pm 1.6$ & $07.1 \pm 1.7$ & $c .065 \pm 0.023$ \\
HI & $0.071 \pm 0.021$ & $0.066 \pm 0.012$ & $0.091 \pm 0.024$ & $0.71 \pm 0.10$ & $b, \mathrm{~d}, f$ \\
Cl & $0.74 \pm 0.10$ & $0.73 \pm 0.10$ & $0.73 \pm 0.13$ & $>0.05$ \\
\hline
\end{tabular}

Each value was calculated from the data for all 10 patients. Values are expressed as mean \pm SD. sIMRT step-and-shoot intensity-modulated radiotherapy, dIMRT dynamic intensity-modulated radiation therapy, VMAT volumetric-modulated arc therapy, Tomo helical tomotherapy. a: sIMRT vs dIMRT, b: sIMRT vs VMAT, c: sIMRT vs Tomo, d: dIMRT vs VMAT, e: dIMRT vs Tomo, f: VMAT vs Tomo. If $c$ is in the "p value" column, it means that there is statistical significance between sIMRT and Tomo 
Table 3 Dose parameters for the PTVbrain according to the four planning modalities

\begin{tabular}{|c|c|c|c|c|c|}
\hline & sIMRT & dIMRT & VMAT & Tomo & $p$ value \\
\hline D2\% (Gy) & $39.1 \pm 1.2$ & $39.4 \pm 1.1$ & $40.6 \pm 2.4$ & $37.5 \pm 2.8$ & $e, f$ \\
\hline D98\% (Gy) & $14.7 \pm 3.2$ & $14.9 \pm 3.3$ & $21.7 \pm 1.1$ & $25.2 \pm 2.0$ & $b, c, d, e, f$ \\
\hline Dmedian (Gy) & $32.1 \pm 0.7$ & $32.1 \pm 0.6$ & $33.2 \pm 1.1$ & $31.6 \pm 0.4$ & $b, d, f$ \\
\hline TC (\%) & $88.2 \pm 4.2$ & $90.6 \pm 1.8$ & $88.7 \pm 2.6$ & $92.6 \pm 2.1$ & $p=0.05$ \\
\hline $\mathrm{HI}$ & $0.76 \pm 0.11$ & $0.76 \pm 0.12$ & $0.57 \pm 0.08$ & $0.39 \pm 0.11$ & $b, c, d, e, f$ \\
\hline $\mathrm{Cl}$ & $0.75 \pm 0.04$ & $0.77 \pm 0.03$ & $0.76 \pm 0.03$ & $0.79 \pm 0.06$ & $>0.05$ \\
\hline
\end{tabular}

Each value was calculated from the data for all 10 patients. Values are expressed as mean $\pm \mathrm{SD}$. sIMRT step-and-shoot intensity-modulated radiotherapy, dIMRT dynamic intensity-modulated radiation therapy, VMAT volumetric-modulated arc therapy, Tomo helical tomotherapy. a: sIMRT vs dIMRT, b: sIMRT vs VMAT, c: sIMRT vs Tomo, d: dIMRT vs VMAT, e: dIMRT vs Tomo, f: VMAT vs Tomo. If $\mathrm{c}$ is in the "p value" column, it means that there is statistical significance between sIMRT and Tomo

lowest average minimum doses (D100\%) to the hippocampus was achieved by Tomo plan.

The entire auditory-vestibular system is vulnerable to RT injury. Therefore, we constrained the inner ear dose to Dmean $\leq 15$ Gy. Table 4 also summarizes the mean inner ear doses for the four plan modalities. There were no significant differences across modalities.

\section{Doses to other OARs}

For eyes, lenses, optic nerves and brainstem, structures not specifically mentioned in the RTOG 0933 protocol dosimetric compliance criteria, all four techniques met the dose constraints we set. The average maximum doses are compared in Table 4. Tomo yielded the lowest average maximum doses to lenses, optic nerves and brainstem. The highest average maximum dose in lenses was achieved by the VMAT plan, and the difference was significant. These differences are attributable to the plans with two full non-coplanar arcs.

\section{Discussion}

WBRT is associated with short local control and side effects, including neurocognition dysfunctions [8] and inner ear deficits [9]. The potential radiation-induced damage to OARs have been underestimated. With the development of sIMRT, dIMRT, VMAT and Tomo, it is feasible to reduce neurocognitive toxicity and inner ear deficits by using advanced techniques. It is possible that SIB-WBRT with hippocampus and inner ear avoidance can achieve the best balance between intracranial tumor

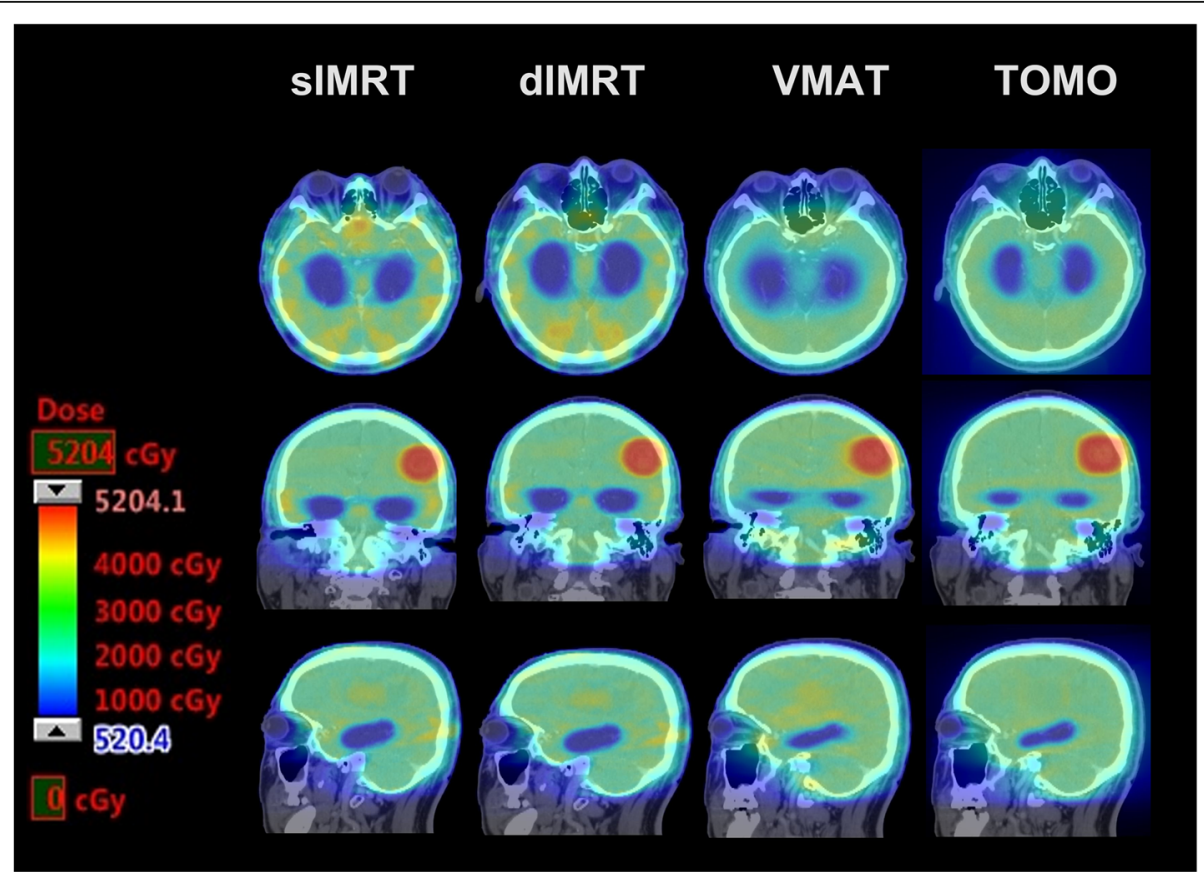

Fig. 1 Color wash comparison of dose distributions for four modalities in a representative patient. sIMRT: step-and-shoot intensity-modulated radiotherapy, dIMRT: dynamic intensity-modulated radiation therapy, VMAT: volumetric-modulated arc therapy, Tomo: helical tomotherapy 


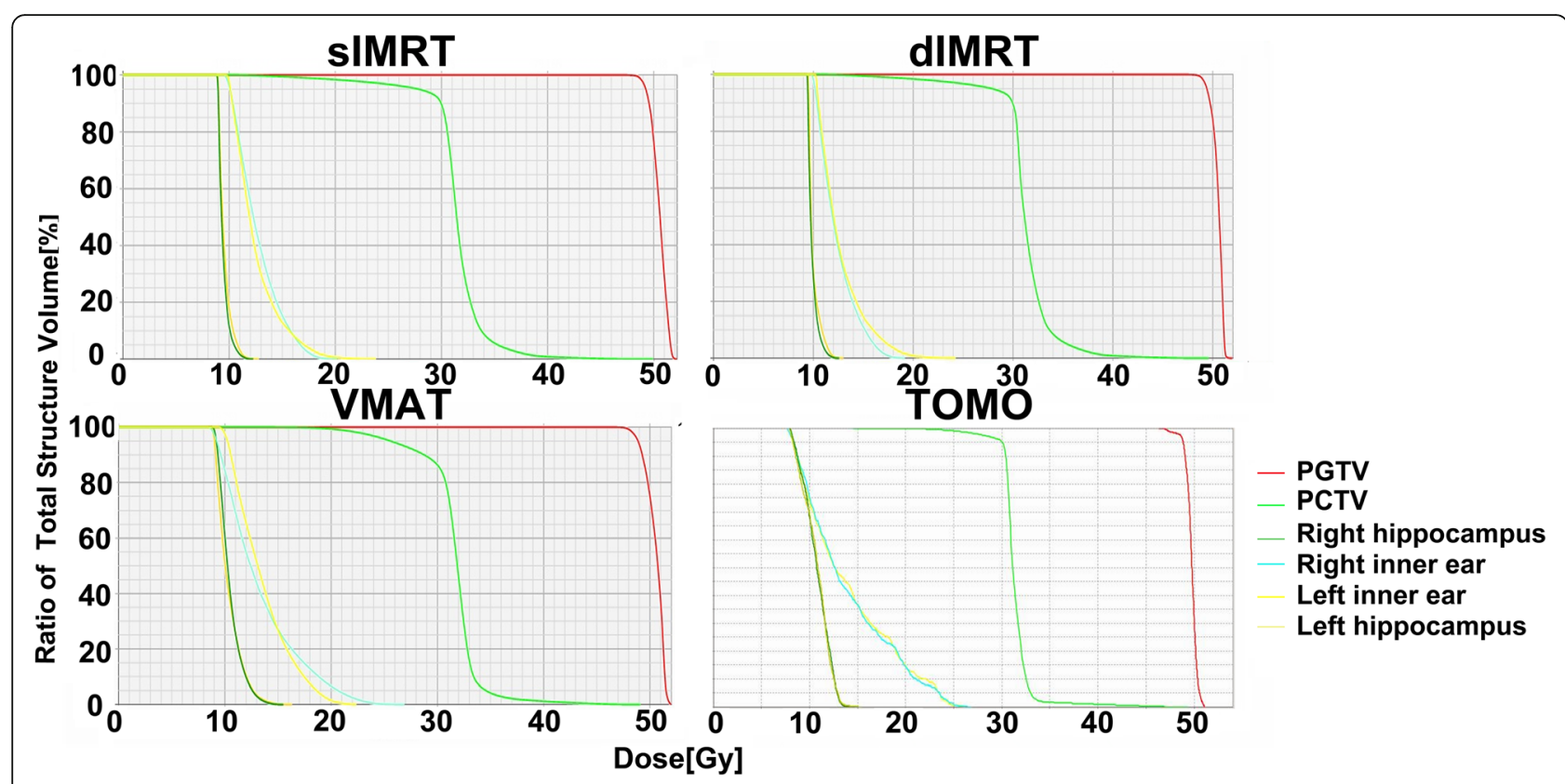

Fig. 2 Dose-volume histograms for treatment planning with the four modalities for a representative patient. sIMRT: step-and-shoot intensity-modulated radiotherapy, dIMRT: dynamic intensity-modulated radiation therapy, VMAT: volumetric-modulated arc therapy, Tomo: helical tomotherapy

control and cognitive function protection. In the present study, we evaluated the dosimetric advantages of four SIB-WBRT radiotherapy modalities (sIMRT, dIMRT, VMAT and Tomo) with hippocampus and inner ear avoidance for the treatment of limited brain metastases. To summarize the results, while all four modalities achieved PTVmets target coverage and met the basic dosimetric compliance criteria, we found that TomoTherapy provided the most homogeneous PTVbrain target dose (mean $\mathrm{HI}=0.39$ ).

Recent reports on SIB-WBRT showed promising outcomes with a shorter treatment period $[15,16]$. Both median intracranial progression-free survival and median survival were increased up to 10 months [24]. The
SIB technique was also found to achieve good hippocampus sparing [25]. In the present study, SIB-WBRT with hippocampus and inner ear avoidance was replanned and compared. All four planning modalities provided good mean PTVmets target prescription coverage (range, 97.1-99.1\%) and similar D2\%, D98\%, Dmedian, and CI values. The HI value for VMAT was higher than those with the other three modalities (0.091 vs $0.065-0.071, p<0.05)$, but this difference is small and has little clinical significance considering that brain metastases are commonly surrounded by healthy brain.

For the whole brain volume, good target coverage, CI and dose homogeneity are very important. In our study, the best target dose homogeneity was achieved by Tomo

Table 4 Doses to OARs

\begin{tabular}{|c|c|c|c|c|c|}
\hline & sIMRT & dIMRT & VMAT & Tomo & $p$ value \\
\hline Hippocampus Dmax (Gy) & $14.3 \pm 1.1$ & $14.5 \pm 1.4$ & $15.8 \pm 0.7$ & $15.5 \pm 0.9$ & $b, c, d, e$ \\
\hline Hippocampus Dmean (Gy) & $9.7 \pm 0.2$ & $10.1 \pm 0.3$ & $10.0 \pm 0.6$ & $10.0 \pm 0.7$ & $>0.05$ \\
\hline Hippocampus Dmin (Gy) & $8.9 \pm 0.3$ & $9.3 \pm 0.2$ & $8.3 \pm 0.4$ & $7.6 \pm 0.7$ & $a, b, c, d, e, f$ \\
\hline Left inner ear Dmean (Gy) & $13.7 \pm 0.7$ & $13.8 \pm 0.8$ & $13.4 \pm 1.0$ & $13.1 \pm 1.0$ & $>0.05$ \\
\hline Right inner ear Dmean (Gy) & $13.6 \pm 0.8$ & $13.8 \pm 0.7$ & $13.7 \pm 1.6$ & $13.3 \pm 1.1$ & $>0.05$ \\
\hline Eyes Dmax (Gy) & $32.5 \pm 4.9$ & $32.9 \pm 4.8$ & $26.7 \pm 6.1$ & $30.8 \pm 4.7$ & $b, d, f$ \\
\hline Lens Dmax (Gy) & $5.7 \pm 0.6$ & $5.8 \pm 0.6$ & $6.8 \pm 1.3$ & $5.5 \pm 0.5$ & $b, d, f$ \\
\hline Optic nerves Dmax (Gy) & $33.6 \pm 1.7$ & $33.8 \pm 1.7$ & $32.0 \pm 2.0$ & $31.3 \pm 0.9$ & $c, e$ \\
\hline Brainstem Dmax (Gy) & $35.4 \pm 1.1$ & $35.7 \pm 0.9$ & $35.9 \pm 0.6$ & $34.0 \pm 0.2$ & $c, e, f$ \\
\hline
\end{tabular}

Each value was calculated from the data of all 10 patients. Values are expressed as mean \pm SD. sIMRT step-and-shoot intenseity-modulated radiotherapy, dIMRT dynamic-intensity modulated radiation therapy, VMAT volumetric-modulated arc therapy, Tomo helical tomotherapy. a: sIMRT vs dIMRT, b: sIMRT vs VMAT, c: sIMRT vs Tomo, d: dIMRT vs VMAT, e: dIMRT vs Tomo, f: VMAT vs Tomo. If $\mathrm{c}$ is in the "p value" column, it means that there is statistical significance between sIMRT and Tomo 
$(0.39, \mathrm{p}<0.05)$, followed by VMAT $(0.57, \mathrm{p}<0.05)$. The two fixed gantry IMRT modalities (step and shot, dynamic) provided similar dose homogeneity (0.76, respectively). Tomo plans could achieve better dose distribution in the PTVbrain over the other techniques, as already confirmed by Cozzi et al. [26] No statistically significant differences in TC and CI were found across modalities. Thus, the four modalities yielded similar results in target volume coverage and conformity.

A phase II multi-institutional trial (RTOG 0933) showed that hippocampus avoidance during WBRT is associated with preservation of memory and quality of life in patients with brain metastases [8]. The development of advanced techniques such as VMAT and Tomo is increasing the feasibility of this treatment paradigm [27-29]. In the present study, we demonstrated that all four planning modalities (sIMRT, dIMRT, VMAT and Tomo) met the RTOG 0933 protocol dose compliance criteria for hippocampal sparing. Similar mean doses to the hippocampi were achieved with the four planning modalities with an acceptable mean PTVbrain coverage (88.2\%-92.6). Compared to healthy brain, the dose to the hippocampi was lower by approximately two-thirds. The entire inner ear system is also vulnerable to RT injury, and dose reduction to this region must be considered even at the relatively low dose of WBRT, as many patients receive some form of cytotoxic therapy. We also demonstrated that the mean inner ear dose was significantly reduced to Dmean $\leq 15 \mathrm{~Gy}$. None of the four modalities produced any notable dose difference in the dose to the brainstem, since the brainstem contour overlapped the PTVbrain volume in all cases. With the use of two full non-coplanar arcs, the average maximum dose to the lenses was highest with VMAT planning.

Although our results show that the four SIB-WBRT modalities can produce acceptable treatment plans with good avoidance of the hippocampus and inner ear, it is important to note that some limitations of the work exsist. For instance, the four investigated techniques are not only delivery techniques but also associated to treatment planning as well, in particular to plan optimization. In addition, treatments using the CyberKnife or GammaKnife are also very common for brain metastases [30, 31], which are not mentioned in the study. Moreover, only one physicist was involved in the treatment planning. This procedure could bring some bias to our result. The application of automatic treatment planning approaches promises to reduce this bias.

\section{Conclusions}

Our data has demonstrated that all four SIB-WBRT modalities (sIMRT, dIMRT, VMAT and Tomo) can produce acceptable treatment plans with good avoidance of the hippocampus and inner ear. Tomo can provide satisfactory PTVbrain coverage with the highest HI but seems the least efficient, whereas VMAT can provide a sufficient dose distribution with remarkably reduced MUs.

\section{Abbreviations}

BED: biological equivalent dose; Cl: conformity index; CT: computed tomography; CTV: clinical target volume; dIMRT: dynamic intensitymodulated radiation therapy; Dmax: maximum dose; Dmax: maximum dose; Dmin: minimum dose; DVH: dose-volume histogram; GTV: gross tumor volume; HI: homogeneity index; MRI: magnetic resonance imaging; MUs: monitor units; NSCLC: non-small cell lung cancer; OARs: dose to organs at risk; OS: overall survival; PTVbrain: planning clinical target volume; PTVmets: planning gross tumor volume; SIB: simultaneous integrated boost; SIMRT: step-and-shoot intensity-modulated radiotherapy; TC: target coverage; Tomo: helical tomotherapy; VMAT: volumetric-modulated arc therapy; WBRT: Whole brain radiotherapy

\section{Acknowledgements}

Not applicable.

\section{Funding}

This study was partially supported by the Shandong Key Research and Development Plan (grant numbers 2017CXGC1209 and 2017GSF18164), the Outstanding Youth Natural Science Foundation of Shandong Province (grant number JQ201423), the Jinan Clinical Medicine Science and Technology Innovation Plan (grant number 201704095), NSFC (grant number 81372413), NSFC (grant number 81671785), the Natural Science Foundation of Shandong Province (grant number 2016ZRC03118), the National Key Research and Development Program of China (grant number 2016YFC0904700), and the Project of Invigorating Health Care through Science, Technology and Education (grant number CXTDA2017034).

\section{Availability of data and materials}

Not applicable.

\section{Authors' contributions}

YSH designed the study; FZ, YQX and WSZ collected the patients' clinical data and delineated target volume; SWP and ZJ optimized and expertreviewed the patients' treatment plans; JAJ analyzed the data and wrote the paper. All authors read and approved the final manuscript.

Ethics approval and consent to participate

The clinical research committee of the study institute approved the study protocol, and written informed consent was waived by the Institutional Review Board

Consent for publication

Not applicable.

\section{Competing interests}

The authors declare that they have no competing interests.

\section{Publisher's Note}

Springer Nature remains neutral with regard to jurisdictional claims in published maps and institutional affiliations.

\section{Author details}

'Shandong University, Jinan 250117, Shandong, China. ${ }^{2}$ Department of Radiation Oncology, Shandong Cancer Hospital affiliated to Shandong University, 440 Jiyan Road, Jinan 250117, Shandong, China.

Received: 10 September 2018 Accepted: 7 March 2019

Published online: 15 March 2019

\section{References}

1. Tsao MN, Xu W, Wong RK, Lloyd N, Laperriere N, Sahgal A, et al. Whole brain radiotherapy for the treatment of newly diagnosed multiple brain metastases. Cochrane Database Syst Rev. 2018. https://doi.org/10.1002/ 14651858. 
2. Soffietti R, Ruda R, Trevisan E. Brain metastases: current management and new developments. Curr Opin Oncol. 2008;20:676-84.

3. Kong W, Jarvis CR, Sutton DS, Ding K, Mackillop WJ. The use of palliative whole brain radiotherapy in the management of brain metastases. Clin Oncol (R Coll Radiol). 2012. https://doi.org/10.1016/j.clon.2012.08.004.

4. Chang EL, Wefel JS, Hess KR, et al. Neurocognition in patients with brain metastases treated with radiosurgery or radiosurgery plus whole-brain irradiation: a randomised controlled trial. Lancet Oncol. 2009:10:1037-44.

5. Rades D, Kueter JD, Hornung D, Veninga T, Hanssens P, Schild SE, et al. Comparison of stereotactic radiosurgery (SRS) alone and whole brain radiotherapy (WBRT) plus a stereotactic boost (WBRT+SRS) for one to three brain metastases. Strahlenther Onkol. 2008;184:55-62.

6. Aoyama H, Shirato H, Tago M, Nakagawa K, Toyoda T, Hatano K, et al. Stereotactic radiosurgery plus whole-brain radiation therapy vs stereotactic radiosurgery alone for treatment of brain metastases: a randomized controlled trial. JAMA. 2006;295:2483-91.

7. Kocher M, Soffietti R, Abacioglu U, Villà S, Fauchon F, Baumert BG, et al. Adjuvant whole-brain radiotherapy versus observation after radiosurgery or surgical resection of one to three cerebral metastases: results of the EORTC 22952-26001 study. J Clin Oncol. 2011;29:134-41.

8. Gondi V, Pugh SL, Tome WA, Caine C, Corn B, Kanner A, et al. Preservation of memory with conformal avoidance of the hippocampal neural stem-cell compartment during whole-brain radiotherapy for brain metastases (RTOG 0933): a phase II multi-institutional trial. J Clin Oncol. 2014;32:3810-6.

9. Hajisafari A, Bakhshandeh M, Aghamiri SMR, Houshyari M, Rakhsha A, Bolokat ER, et al. Prospective evaluation of the early effects of radiation on the auditory system frequencies of patients with head and neck cancers and brain tumors after radiotherapy. Ear Nose Throat J. 2018;97:E10-7.

10. Abayomi OK. Pathogenesis of irradiation-induced cognitive dysfunction. Acta Oncol. 1996;35:59-63.

11. Tang FR, Loke WK, Khoo BC. Postnatal irradiation-induced hippocampal neuropathology, cognitive impairment and aging. Brain and Development. 2017:39:277-93.

12. Ji S, Tian Y, Lu Y, Sun R, Ji J, Zhang L, et al. Irradiation-induced hippocampal neurogenesis impairment is associated with epigenetic regulation of bdnf gene transcription. Brain Res. 2014;1577:77-88.

13. Tan PX, Du SS, Ren C, Yao QW, Yuan YW. Radiation-induced cochlea hair cell death: mechanisms and protection. Asian Pac J Cancer Prev. 2013;14:5631-5

14. Hitchcock YJ, Tward JD, Szabo A, Bentz BG, Shrieve DC. Relative contributions of radiation and cisplatin-based chemotherapy to sensorineural hearing loss in head-and-neck cancer patients. Int J Radiat Oncol Biol Phys. 2009;73:779-88.

15. Dobi Á, Fodor E, Maráz A, Együd Z, Cserháti A, Tiszlavicz L, et al. Boost Irradiation Integrated to Whole Brain Radiotherapy in the Management of Brain Metastases. Pathol Oncol Res. 2018. https://doi.org/10.1007/s12253-018-0383-y.

16. Rodrigues G, Yartsev S, Tay KY, Pond GR, Lagerwaard F, Bauman G. A phase II multi-institutional study assessing simultaneous in-field boost helical tomotherapy for 1-3 brain metastases. Radiat Oncol. 2012;7:42.

17. Ferro M, Cilla S, Macchia G, Deodato F, Pierro A, Digesu' C, et al. On the cutting edge of intensity modulated radiotherapy and simultaneous integrated boost (IMRT-SIB): the case of a patient with 8 brain metastases. Rep Pract Oncol Radiother. 2015;20(4):316-9.

18. Sood S, Pokhrel D, McClinton C, Lominska C, Badkul R, Jiang H, et al. Volumetric-modulated arc therapy (VMAT) for whole brain radiotherapy: not only for hippocampal sparing, but also for reduction of dose to organs at risk. Med Dosim. 2017;42:375-83.

19. Gutiérrez AN, Westerly DC, Tomé WA, Jaradat HA, Mackie TR, Bentzen SM, et al. Whole brain radiotherapy with hippocampal avoidance and simultaneously integrated brain metastases boost: a planning study. Int J Radiat Oncol Biol Phys. 2007;69:589-97.

20. Tsao MN, Lloyd N, Wong RK, Chow E, Rakovitch E, Laperriere N, et al. Whole brain radiotherapy for the treatment of newly diagnosed multiple brain metastases. Cochrane Database Syst Rev. 2012. https:/doi.org/10.1002/14651858.

21. Barendsen GW. Dose fractionation, dose rate and iso-effect relationships for normal tissue responses. Int J Radiat Oncol Biol Phys. 1982:8:1981-97.

22. Paddick I. A simple scoring ratio to index the conformity of radiosurgical treatment plans. Technical note. J Neurosurg. 2000;93(Suppl 3):219-22.

23. Kataria T, Sharma K, Subramani V, Karrthick KP, Bisht SS. Homogeneity index: an objective tool for assessment of conformal radiation treatments. J Med Phys. 2012;37:207-13.
24. Zhou L, Liu J, Xue J, Xu Y, Gong Y, Deng L, et al. Whole brain radiotherapy plus simultaneous in-field boost with image guided intensity-modulated radiotherapy for brain metastases of non-small cell lung cancer. Radiat Oncol. 2014:9:117.

25. Prokic V, Wiedenmann N, Fels F, Schmucker M, Nieder C, Grosu AL. Whole brain irradiation with hippocampal sparing and dose escalation on multiple brain metastases: a planning study on treatment concepts. Int J Radiat Oncol Biol Phys. 2013;85:264-70.

26. Cozzi L, Clivio A, Bauman G, Cora S, Nicolini G, Pellegrini R, et al. Comparison of advanced irradiation techniques with photons for benign intracranial tumours. Radiother Oncol. 2006;80:268-73.

27. Kothavade V, Jamema SV, Gupta T, Pungavkar S, Upasani M, Juvekar S, et al. Which is the most optimal technique to spare hippocampus?Dosimetric comparisons of SCRT, IMRT, and tomotherapy. I Cancer Res Ther. 2015:11:358-63.

28. Rong Y, Evans J, Xu-Welliver M, Pickett C, Jia G, Chen Q, et al. Dosimetric evaluation of intensity-modulated radiotherapy, volumetric modulated arc therapy, and helical tomotherapy for hippocampal-avoidance whole brain radiotherapy. PLoS One. 2015. https://doi.org/10.1371/journal.pone.0126222.

29. Pokhrel D, Sood S, Lominska C, Kumar P, Badkul R, Jiang H, et al. Potential for reduced radiation-induced toxicity using intensity-modulated arc therapy for whole-brain radiotherapy with hippocampal sparing. J Appl Clin Med Phys. 2015;16:131-41

30. Liu SH, Murovic J, Wallach J, Cui G, Soltys SG, Gibbs IC, et al. CyberKnife radiosurgery for brainstem metastases: management and outcomes and a review of the literature. J Clin Neurosci. 2016;25:105-10.

31. Yamamoto M, Serizawa T, Higuchi Y, Sato Y, Kawagishi J, Yamanaka K, et al. A multi-institutional prospective observational study of stereotactic radiosurgery for patients with multiple brain metastases (JLGK0901 study update): irradiation-related complications and long-term maintenance of mini-mental state examination scores. Int J Radiat Oncol Biol Phys. 2017;99:31-40.

Ready to submit your research? Choose BMC and benefit from:

- fast, convenient online submission

- thorough peer review by experienced researchers in your field

- rapid publication on acceptance

- support for research data, including large and complex data types

- gold Open Access which fosters wider collaboration and increased citations

- maximum visibility for your research: over $100 \mathrm{M}$ website views per year

At $\mathrm{BMC}$, research is always in progress.

Learn more biomedcentral.com/submissions 